\title{
Iron-dependent CDK1 activity promotes lung carcinogenesis via activation of the GP130/STAT3 signaling pathway
}

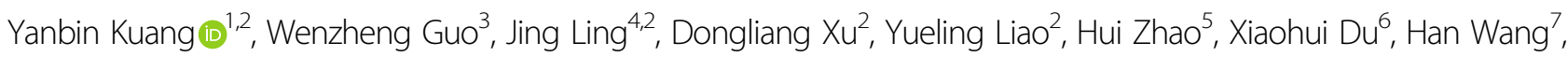
Mingxin Xu${ }^{1}$, Hongyong Song ${ }^{2}$, Tong Wang ${ }^{2}$, Bo Jing ${ }^{2}$, Kaimi $\mathrm{Li}^{2}$, Min $\mathrm{Hu}^{2}$, Wenjuan $\mathrm{Wu}^{3}$, Jiong Deng ${ }^{2}$ and Qi Wang ${ }^{1}$

\begin{abstract}
Iron dysregulation is associated with several diseases, including lung cancer, but the underlying mechanism is yet unknown. Iron directly binds CDK1, which is upregulated in several cancers, thereby promoting JAK1 phosphorylation and activation of STAT3 signaling to promote colorectal carcinogenesis. This study aimed to investigate the role of iron/CDK1/STAT3 signaling in lung carcinogenesis. We found that iron-dependent CDK1 activity upregulated IL-6 receptor subunit GP130 post-transcriptionally via phosphorylation of 4E-BP1, which is critical for activation of JAK/ STAT3 signaling. CDK1 and STAT3 are essential for iron-mediated colony formation in lung cancer cell lines. CDK1 knockdown and iron chelator DFO decreased tumorigenicity and GP130/STAT3 signaling in vivo. Moreover, CDK1/ GP130/STAT3 signaling were elevated in lung cancer tissues compared with adjacent normal lung tissues. Altogether, the present results suggest that CDK1 inhibition and iron deprivation are potential strategies to target GP130/ STAT3 signaling to suppress lung cancer.
\end{abstract}

\section{Introduction}

Lung cancer is the second most common cancer and the leading cause of cancer-related mortality among men and women worldwide ${ }^{1}$; however, its underlying mechanism remains unclear. Hence, a better understanding of the molecular mechanism underlying lung carcinogenesis would contribute to the development of novel strategies for its prevention and targeted therapy.

Cyclin-dependent kinases (CDKs) are critical drivers of cell cycle transition. In particular, CDK1 is a key determinant of mitotic progression, and its activity is dysregulated via indirect genetic alteration in tumorigenesis ${ }^{2}$.

\footnotetext{
Correspondence: Jiong Deng (jiongdeng@shsmu.edu.cn) or Qi Wang (wqdlmu@163.com)

${ }^{1}$ Department of Respiratory Medicine, The Second Affiliated Hospital, Dalian Medical University, Dalian, China

${ }^{2}$ Key Laboratory of Cell Differentiation and Apoptosis of Chinese Minister of Education, Shanghai, China

Full list of author information is available at the end of the article.

These authors contribute equally: Yanbin Kuang, Wenzheng Guo, Jing Ling Edited by G. Ciliberto
}

Integration of gene expression data from different databases (TCGA and GEO) demonstrated CDK1 upregulation in lung adenocarcinoma. Furthermore, CDK1 upregulation is associated with a poor prognosis ${ }^{3}$. Selective targeting of CDK1 might constitute a novel strategy for tumor treatment in certain contexts. CDK1 inhibition is selectively lethal in MYC-dependent human breast cancer cells ${ }^{4}$. As CDK1 activity is essential for the formation of BRCA-1 foci, its inhibition, combined with PARP inhibition, could effectively reduce BRCAproficient tumor growth and regression ${ }^{5}$. CDK1 inhibition has reportedly increased the efficacy of sorafenib in hepatocellular carcinoma $^{6}$. However, the molecular mechanisms and potential applications of CDK1 in lung cancer remain undetermined.

Iron is an essential nutrient, and its excess or deficient levels may affect diverse biological processes ${ }^{7}$. Altered iron metabolism is closely associated with cancer initiation and progression. Epidemiological studies have reported that high-dietary iron intake significantly elevate

\section{(c) The Author(s) 2019}

(c) (i) Open Access This article is licensed under a Creative Commons Attribution 4.0 International License, which permits use, sharing, adaptation, distribution and reproduction cc) in any medium or format, as long as you give appropriate credit to the original author(s) and the source, provide a link to the Creative Commons license, and indicate if changes were made. The images or other third party material in this article are included in the article's Creative Commons license, unless indicated otherwise in a credit line to the material. If material is not included in the article's Creative Commons license and your intended use is not permitted by statutory regulation or exceeds the permitted use, you will need to obtain permission directly from the copyright holder. To view a copy of this license, visit http://creativecommons.org/licenses/by/4.0/. 
the risk of several malignancies, including esophageal cancer, colorectal cancer, liver cancer, and lung cancer ${ }^{8}$. Consistent with epidemiological data, experimental studies have reported that a high-iron diet promotes spontaneous colorectal tumorigenesis and mammary tumor growth in mice ${ }^{9,10}$. On the contrary, a low-iron diet reportedly decreased tumor growth in mice ${ }^{11}$. Iron chelators inhibit human lung tumor xenografts ${ }^{12}$. In addition, a low-iron diet or the use of iron chelators protected cigarette-smoke-induced chronic obstructive pulmonary disease $(\mathrm{COPD})^{13}$, which is related to 4.5 -fold higher incidence of lung cancer than in the general population ${ }^{14}$. Altogether, these studies suggested the important role of iron in lung carcinogenesis; however, the underlying molecular mechanism remains unknown. Recently, iron was reported to bind CDK1 directly and enhance its activity. CDK1 phosphorylates JAK1 to activate the JAK/ STAT3 signaling pathway and promote colorectal tumorigenesis 9 . However, in the presence of CDK1, a combination of IL- 6 and iron elevated STAT3 activity to a greater extent than iron alone ${ }^{9}$. This implies the existence of the yet unrevealed mechanism underlying irondependent CDK1-mediated STAT3 activation, such as the regulation of IL- 6 receptor subunit GP130 by irondependent CDK1 activity.

GP130 is the central player of the receptor complexes formed by IL- 6 cytokines. When IL- 6 binds IL-6R $\alpha$, GP130 dimerizes and activates JAK/STAT3 signaling ${ }^{15}$. IL-6 and related cytokines are critical lynchpins between inflammation and cancer ${ }^{16}$. Systemic and pulmonary IL-6 levels are reportedly elevated in patients with lung adenocarcinoma and these elevations are associated with poor patient survival ${ }^{17-20}$. IL-6 promotes KRAS-driven lung carcinogenesis, and genetic ablation of either IL-6 or STAT3 suppresses the extent of lung cancer ${ }^{21}$. Moreover, blockade of IL-6/STAT3 axis reportedly suppressed cachexia in the same model ${ }^{22}$. These evidences above suggest that regulation of GP130 might suppress lung cancer.

In this study, we investigated the association among iron, CDK1, and GP130/STAT3 signaling in lung carcinogenesis. Our results may contribute to the development of potential strategies to target GP130/STAT3 signaling and suppress lung cancer via CDK1 blockade and iron deprivation.

\section{Results}

\section{CDK1 and STAT3 are essential for iron-mediated colony} formation in lung cancer cell lines

To investigate whether iron promotes lung carcinogenesis, we first performed a soft-agar colony formation assay and a sphere culture assay using lung adenocarcinoma cell lines A549 and 1792. As expected, addition of ferrous sulfate $(\mathrm{FS}, 100 \mu \mathrm{M})$ (Sigma-Aldrich) in RPMI
1640 culture medium significantly increased the number of colonies in both lung cancer cell lines (Fig. 1a). We further seeded lung cancer cells into low-adherent 96-well plates, cultured them with different conditional media for 12 days, and then observed the size of the spheres. Larger spheres were observed in the FS $(100 \mu \mathrm{M})$ group, which was reversed upon addition of an iron chelator deferoxamine (DFO; $100 \mu \mathrm{M})$. Even a low DFO concentration $(10 \mu \mathrm{M})$ suppressed sphere formation in the control group (not treated with FS in the culture medium) (Fig. 1b).

Iron reportedly binds CDK1 directly and enhances its kinase activity. Iron-dependent CDK1 activity promotes colorectal carcinogenesis via the JAK/STAT3 signaling pathway ${ }^{9}$. To investigate whether CDK1 and STAT3 are essential for iron-mediated colony formation in lung cancer cell lines, we cultured lung cancer cells in lowadherent 96-well plates with RPMI 1640 supplemented with 0 or $100 \mu \mathrm{M} \mathrm{FS}$, and treated the cells with CDK1selective inhibitor RO-3306 (CDK1i, $10 \mu \mathrm{M})$ and STAT3selective inhibitor Stattic $(5 \mu \mathrm{M})$ for 12 days. Both CDK1 and STAT3 inhibition significantly decreased sphere formation, regardless of ferrous sulfate addition (Fig. 1b and Supplementary Fig. 1A). We further used shRNA against CDK1 and STAT3, and established corresponding cell lines with a stable knockdown (Fig. 1c). Knockdown of either CDK1 or STAT3 blocked iron-mediated colony formation (Fig. 1d-f).

\section{Iron-dependent CDK1 activity promotes STAT3 signaling via upregulation of GP130}

It was previously reported that CDK1 phosphorylates JAK1 directly and activates downstream STAT3 signaling. However, from previous work, IL-6 seemed to increase the effect on STAT3 activation'. We speculate that GP130, an IL-6 receptor and upstream receptor of JAK/ STAT3 signaling, might also be regulated by irondependent CDK1 activity. To verify our speculation, we performed western blot analysis to assess GP130 expression and STAT3 activation under different conditions. Iron upregulated GP130 and activated STAT3, whereas CDK1i significantly downregulated GP130 and inactivated STAT3 regardless of FS addition (Fig. 2a, b, Supplementary Fig. 1C, and 1D). Consistent with the data on CDK1i, siRNA-mediated knockdown of CDK1 downregulated GP130 and iron-mediated STAT3 activation (Fig. 2c, d). To further verify GP130-mediated STAT3 activation, we knocked down GP130 and observed a reduction in STAT3 activation regardless of FS addition (Fig. 2c, d). JAK1/2 inhibitor Ruxolitinib (JAKi, $3 \mu \mathrm{M}$ ) robustly blocked JAK/STAT3 signaling; however, it did not affect GP130 expression, indicating that GP130 upregulation was not induced by JAK/STAT3 downstream signaling (Fig. 2e). We further treated lung cancer cells with IL-6 $(10 \mathrm{ng} / \mathrm{ml})$ in the presence of CDK1i or not, and found 

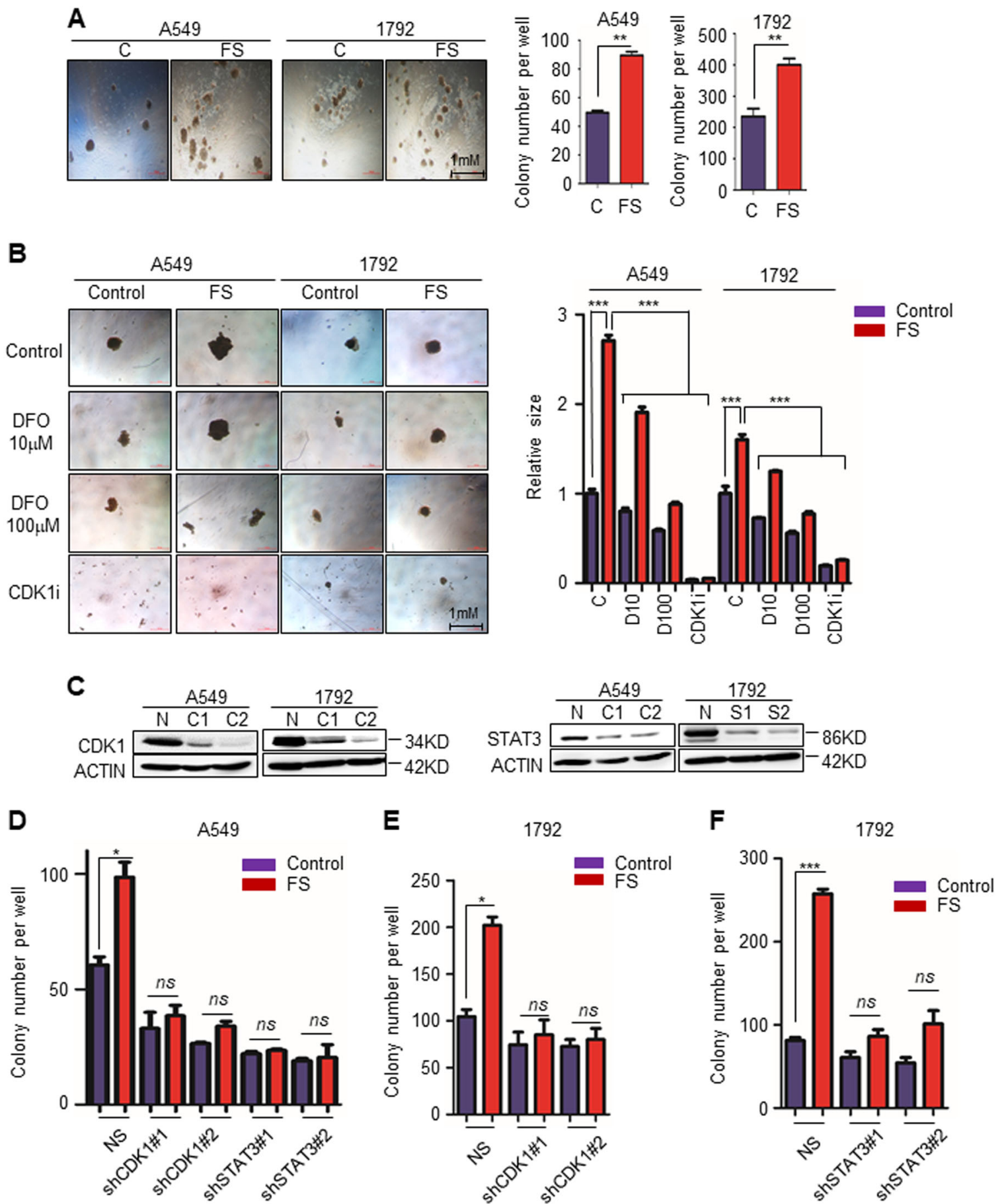

Fig. 1 CDK1 and STAT3 are essential for iron-mediated colony formation in lung cancer cell lines. a Representative bright-field images and quantification of colonies of A549 or 1792 cells per well in RPMI 1640 medium supplemented with 0 (C) or $100 \mu$ M ferrous sulfate (FS) in a soft-agar colony formation assay. b Representative bright-field images and quantification of the sphere size in sphere culture assay. A549 or 1792 cells cultured in RPMI 1640 supplemented with 0 (Control) or $100 \mu \mathrm{M}$ ferrous sulfate (FS), treated with dimethyl sulfoxide (Control), iron chelator DFO (10 $\mu \mathrm{M}$ or $100 \mu \mathrm{M})$, and CDK1-selective inhibitor RO-3306 (CDK1i; $10 \mu \mathrm{M})$. c Western blot analysis for CDK1 or STAT3 expression in A549 and 1792 expressing shRNA against CDK1 (C1 and C2), STAT3 (S1 and S2) or non-specific shRNA NS(N). $\mathbf{d}$-f $\mathbf{f}$ Quantification of colonies per well of the indicated cell lines in RPMI 1640 medium supplemented with 0 (Control) or $100 \mu \mathrm{M}$ ferrous sulfate (FS). ${ }^{*} p<0.05,{ }^{* *} p<0.01,{ }^{* * *} p<0.001$. Error bars represent the SEM values 
A

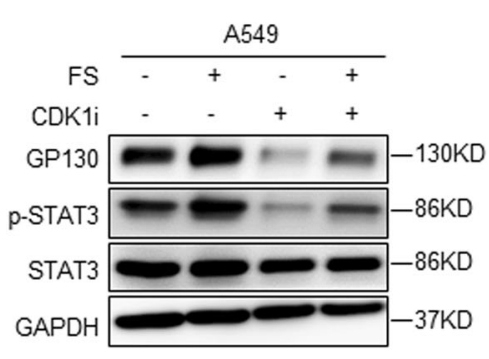

B

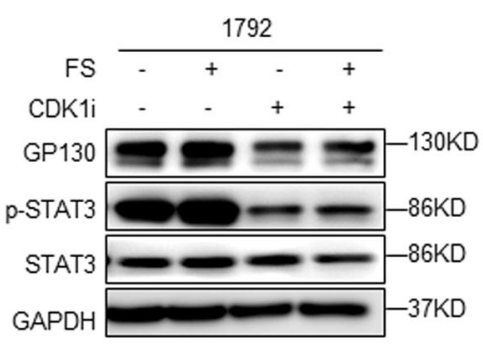

E

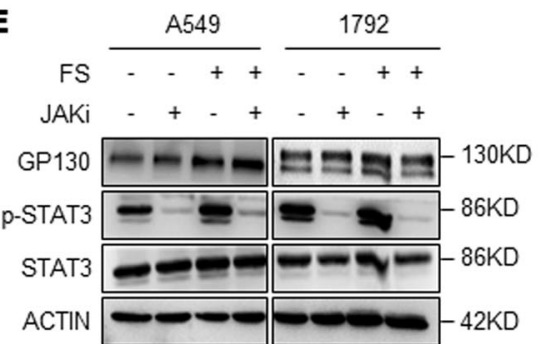

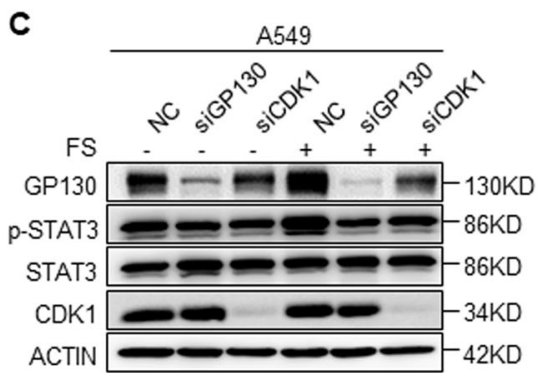

D

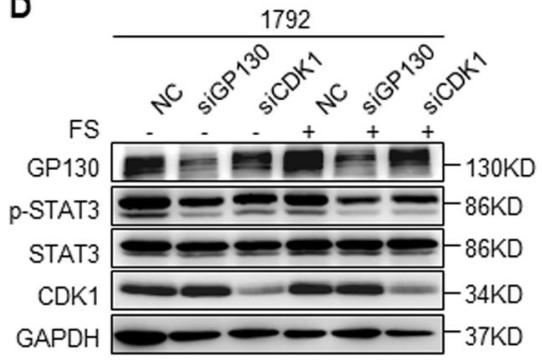

$\mathbf{F}$

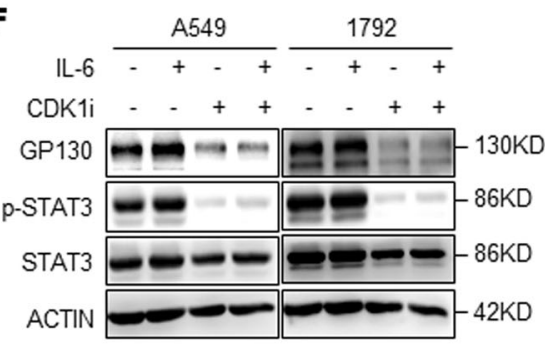

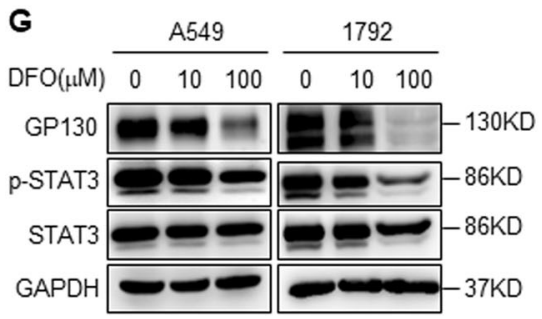

Fig. 2 Iron-dependent CDK1 promotes STAT3 signaling via upregulation of GP130 a b Western blot analysis for GP130, p-STAT3(Y705), and STAT3 expression in A549 or 1792 cells treated with ferrous sulfate (FS; $100 \mu \mathrm{M})$ and CDK1i $(10 \mu \mathrm{M})$ for $24 \mathrm{~h}$. c, d Western blot analysis for GP130, p-STAT3(Y705), STAT3, and CDK1 expression in A549 or 1792 cells transfected with siNC (non-specific control)/siGP130/siCDK1, treated with 0 or

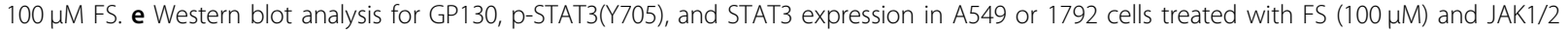
inhibitor Ruxolitinib (JAKi, $3 \mu \mathrm{M}$ ) for $24 \mathrm{~h}$. JAK inhibition decreased STAT3 signaling; however, it did not affect GP130 expression. $\mathbf{f}$ Western blot analysis for GP130, p-STAT3(Y705), and STAT3 expression in A549 or 1792 cells treated with IL-6 (10 ng/ml) and CDK1i (10 $\mu$ M) for $24 \mathrm{~h}$. g Western blot analysis for GP130, p-STAT3(Y705), and STAT3 expression in A549 or 1792 cells treated with iron chelator DFO (10 or $100 \mu \mathrm{M})$ for $24 \mathrm{~h}$

CDK1i downregulated GP130 and blocked STAT3 phosphorylation as well (Fig. 2f).

Furthermore, we treated lung cancer cells with different concentrations of iron chelator DFO $(10 \mu \mathrm{M}$ or $100 \mu \mathrm{M})$. As expected, GP130 was downregulated and STAT3 phosphorylation was decreased upon iron chelation at different degrees (Fig. 2g). Iron increased reactive oxygen species (ROS) levels via the Fenton reaction and oxidative stress triggered STAT3 signaling ${ }^{23,24}$. To exclude the possibility that oxidative stress activates STAT3 signaling and promotes sphere formation directly in our study, we treated lung cancer cells with the antioxidant butylated hydroxyanisole (BHA, $100 \mu \mathrm{M}$ ). However, it failed to suppress sphere formation and ironmediated GP130/STAT3 signaling (Supplementary Fig. 1A, B). 

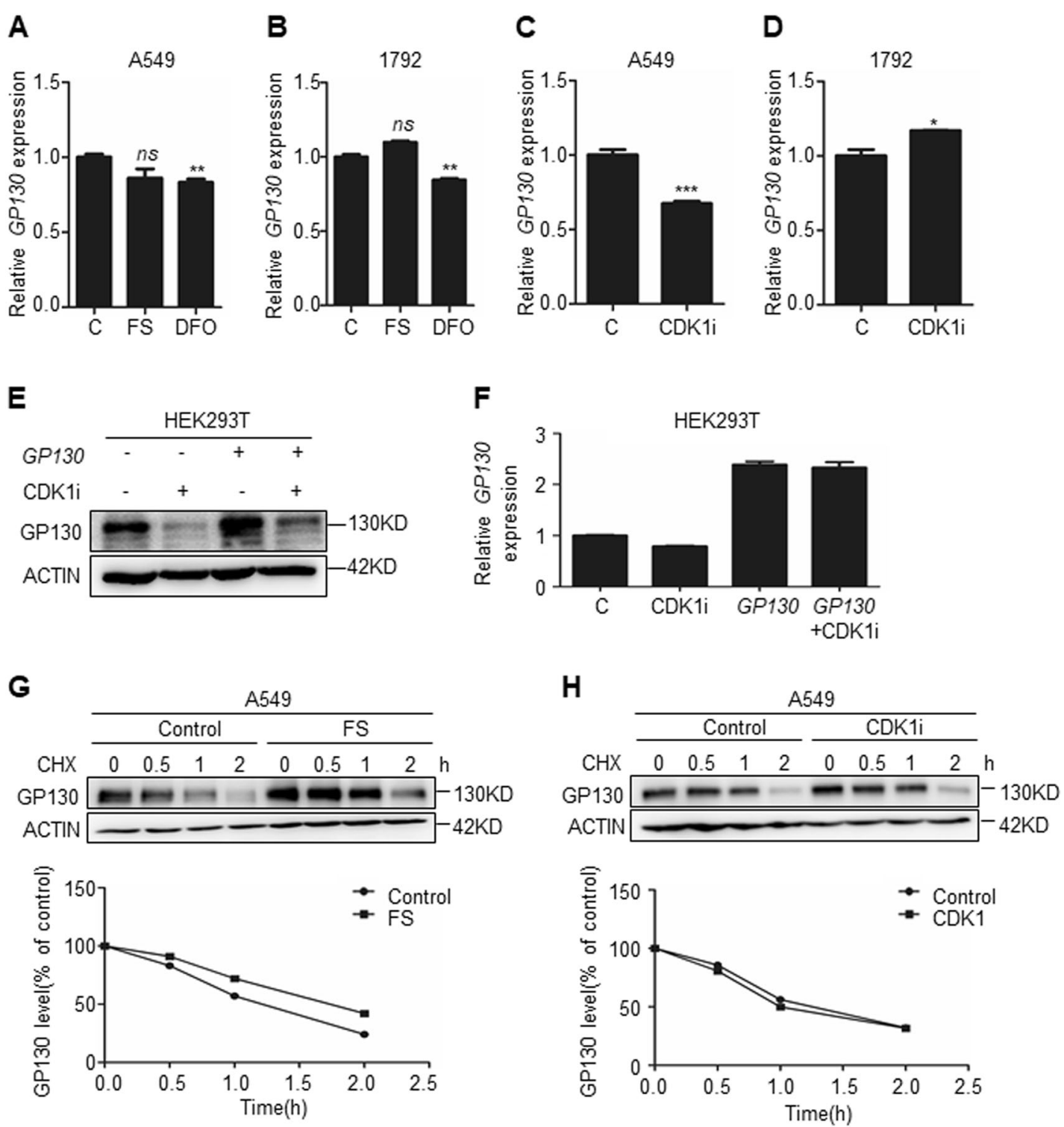

Fig. 3 Iron-dependent CDK1 post-transcriptionally upregulates GP130. a-d The relative GP130 mRNA expression quantified via quantitative polymerase chain reaction (qPCR) for the indicated cell lines treated with ferrous sulfate (FS; $100 \mu \mathrm{M})$ or iron chelator DFO $(100 \mu \mathrm{M})$ or CDK1i (10 $\mu \mathrm{M})$ or untreated control (C) for $24 \mathrm{~h}$. $(n=3)$. e Western blot analysis for GP130 expression in the HEK293T cell line transfected with GP130 or vector control, treated with 0 or $10 \mu \mathrm{M}$ CDK1i for $24 \mathrm{~h}$. f Relative GP130 mRNA expression quantified via qPCR in HEK293T cell line transfected with GP130 or vector control, treated with 0 or $10 \mu \mathrm{M}$ CDK1i for $24 \mathrm{~h}$. $(n=3)$. $\mathbf{g}$, $\mathbf{h}$ Western blot analysis for GP130 expression in A549 cells and the relative amounts of GP130 were calculated after normalization (GP130/ACTIN). At the beginning of each chase, cycloheximide (CHX, $30 \mu \mathrm{g} / \mathrm{ml}$ ) was added to block translation, along with CDK1i $(0$ or $10 \mu \mathrm{M})$. FS $(0$ or $100 \mu \mathrm{M})$ pre-treatment was administered for $24 \mathrm{~h}$. Cells were harvested at the indicated time point. ${ }^{*} p<0.05,{ }^{* *} p<0.01,{ }^{* * *} p<0.001$. Error bars represent the SEM values

\section{Iron-dependent CDK1 activity post-transcriptionally upregulates GP130}

To understand how iron-mediated CDK1 upregulates GP130, we initially quantified GP130 mRNA levels via quantitative real-time polymerase chain reaction (qPCR) under different conditions. GP130 mRNA levels did not increase significantly upon treatment with FS. GP130 mRNA showed in both A549 and 1792 a slight but statistically significant decrease upon treatment with the iron chelator DFO (Fig. 3a, b), suggesting that treatment with this agent might affect to a small extent the transcriptional regulation of GP130. CDK1i partially downregulated GP130 mRNA in A549 cells (Fig. 3c), but it upregulated GP130 mRNA in 1792 cells (Fig. 3d), suggesting that the possible contribution of CDK1 to GP130 mRNA regulation could differ depending on the context. 
To determine whether CDK1 affects GP130 protein level via regulation of GP130 transcription, we overexpressed GP130 in the HEK239T cell line via transfection of the GP130 plasmid, which maintained upregulated GP130 mRNA. Thereafter, these cells were treated with CDK1i for $24 \mathrm{~h}$, and GP130 protein was significantly downregulated regardless of GP130 mRNA expression (Fig. 3e, f).

We further analyzed whether GP130 protein stabilization was regulated by iron-dependent CDK1 activity. At the beginning of each chase, cycloheximide (CHX, $30 \mu \mathrm{g} /$ $\mathrm{ml})$ was added to block translation. CDK1i $(10 \mu \mathrm{M})$ was added at the beginning of each chase. FS $(100 \mu \mathrm{M})$ pretreatment was administered for $24 \mathrm{~h}$. Cells were harvested at the indicated time point. Iron or CDK1i did not affect the rate of GP130 degradation a lot (Fig. 3g, h). We further added MG132 and CHL to inhibit proteasomedependent and lysosome-dependent degradation; however, these inhibitors did not reverse CDK1i- or siCDK1-mediated GP130 downregulation (Supplementary Fig. 2A-D). Altogether, these findings suggest that iron-dependent CDK1 activity upregulates GP130 posttranscriptionally.

\section{Iron-dependent CDK1 activity promotes GP130 cap- dependent translation via phosphorylation of 4E-BP1}

CDK1 reportedly activates cap-dependent translation by directly hyperphosphorylating 4E-BP1 ${ }^{25,26}$. Hypophosphorylated 4E-BP1 could sequester eIF4E from the eIF4F cap initiation complex, but when 4E-BP1 is hyperphosphorylated, it lost this ability to bind eIF4E. Furthermore, hypophosphorylated 4E-BP1 is degraded, while hyperphosphorylated 4E-BP1 is refractory to degradation ${ }^{27}$. Previous studies classified 4E-BP1 into four groups according to its molecular weight and named $\alpha, \beta, \gamma$, and $\delta$. CDK1 phosphorylates 4E-BP1 at T37, T46, S65, T70, and S83 site, resulting in a mitosis-specific hyperphosphorylated $\delta$ isoform $^{25,26}$. However, we notice that mutation of 4E-BP1 at S83 site didnot affect general cap-dependent translation initiation complex formation ${ }^{26}$. This result might suggest that the key point of translation initiation complex formation is more likely 4E-BP1 hyperphosphorylation than $4 \mathrm{E}$ BP1 phosphorylated at specific sites. Therefore, we speculate that iron-dependent CDK1 activity upregulates GP130 via a 4E-BP1 hyperphosphorylation mechanism. To confirm whether GP130 protein synthesis is cap-dependent, we used cap-dependent translation inhibitor 4E1RCat, which prevents eIF4G from binding eIF4E, similar to the role of 4EBP1. 4E1RCat markedly downregulated GP130 regardless of addition of iron (Fig. 4a, b). Thereafter, we investigated whether iron and CDK1 are correlated with 4E-BP1 phosphorylation. A549 and 1792 cells were treated with FS $(100 \mu \mathrm{M})$ and CDK1i $(10 \mu \mathrm{M})$ for $24 \mathrm{~h}$, and iron promoted 4E-BP1 phosphorylation at Ser65/Thr70, which was reversed by CDK1i (Fig. 4c). Consistent with the data on CDK1i, siRNA-mediated knockdown of CDK1 decreased GP130 expression and inhibited 4E-BP1 phosphorylation (Fig. 4d). We treated lung A549 cells with CDK1i for different time and found decreased GP130, p-STAT3, and p4E-BP1 expression along with time (Fig. 4e). To further confirm our speculation, we performed an in vitro phosphorylation assay, and we found that iron promoted 4E-BP1 phosphorylation via CDK1 (Fig. 4f). Furthermore, in vitro protein translation assay was performed with $\mathrm{TnT}^{\oplus}$ Quick Coupled Transcription/Translation System (L1170; Promega). 4E-BP1 inhibited protein translation, but CDK1/ CyclinB1 in the presence of 0 or $10 \mu \mathrm{M}$ FS phosphorylate $4 \mathrm{E}-\mathrm{BP} 1$ and increase protein translation at different degrees (Fig. 4f, g). Therefore, we proposed that hypophosphorylated 4E-BP1 sequesters eIF4E from the eIF4F cap initiation complex and turns GP130 cap-dependent translation "Off." However, when iron binds CDK1 and enhances its activity, is hyperphosphorylates 4E-BP1 and turns capdependent translation "On" (Fig. 4h).

\section{CDK1 knockdown and iron chelator DFO decrease tumorigenicity and GP130/STAT3 signaling in vivo}

To verify whether CDK1 is essential for tumorigenicity in vivo, we established A549-NS, A549-shCDK1\#1 and A549-shCDK1\#2 cell lines using shRNA against CDK1 or NS (non-specific shRNA sequence). These cell lines were injected subcutaneously into nude mice. The tumorigenicity of A549-shCDK1\#2 was significantly lower than that of A549-NS, while that of A549-shCDK1\#1 was slightly but not significantly lower than that of A549-NS (Fig. 5a). This may have resulted from minor differences in CDK1 expression between A549-NS and A549shCDK1\#1 (Fig. 5b). Concurrent with our in vitro data, immunohistochemistry staining of xenograft tissue slides indicated GP130 and p-STAT3 downregulation upon CDK1 knockdown (Fig. 5b).

Furthermore, nude mice were injected with A549 cells $\left(1.5 \times 10^{6}\right.$ cells $)$. After 19 days, the mice were administered an intraperitoneal injection of DFO $(16 \mathrm{mg} / \mathrm{kg})$ dissolved in $0.9 \% \mathrm{NaCl}$, or saline solution alone, once a day for 12 days. DFO significantly decreased tumorigenicity and the expression of GP130 and p-STAT3 (Fig. 5c, d).

\section{CDK1/GP130/STAT3 signaling were greater in lung cancer} tissues than in adjacent normal lung tissues

To further investigate the clinical relevance of our findings, we performed immunohistochemical staining to assess the CDK1, GP130, and p-STAT3 expression in lung cancer tissues and adjacent normal lung tissues. CDK1, GP130, and p-STAT3 were upregulated in lung cancer tissues compared with adjacent normal lung tissues (Fig. 6a, b). We further stained tissue microarrays comprising 36 pairs of lung cancer tissues and adjacent 
A

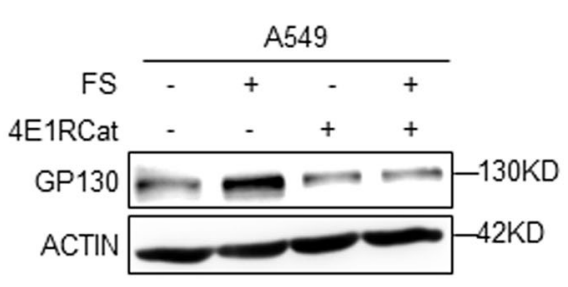

C

FS $\frac{\text { A549 }}{-+-+t} \frac{1792}{-++}$

CDK1i

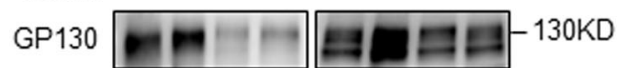

$\mathrm{p}-4 \mathrm{E}-\mathrm{BP} 1 \mathrm{~A}$
(T70)

$\mathrm{p}-4 \mathrm{E}-\mathrm{BP} 1 \mathrm{C}-15 \mathrm{KD}$
$(\mathrm{S} 65)$

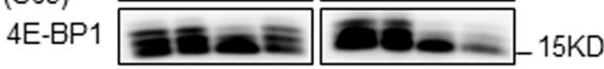

ACTIN

E

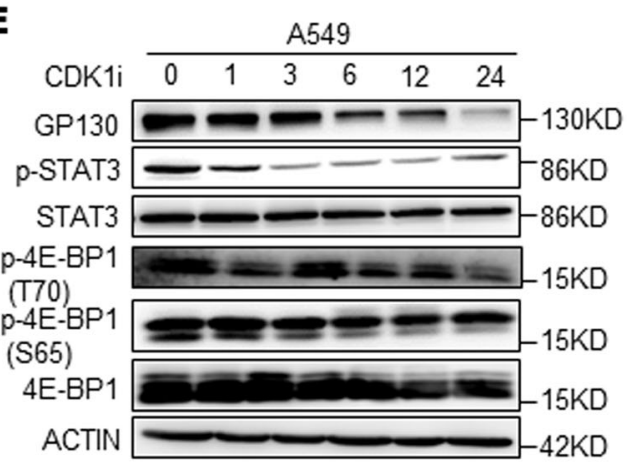

H

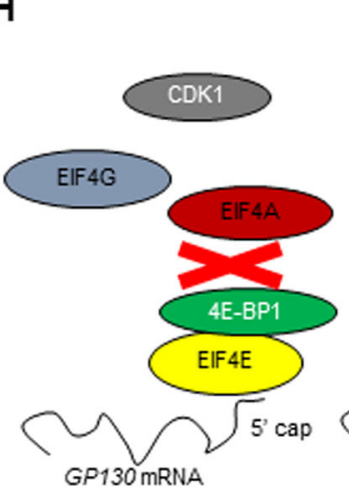

Translation "Off'"
B

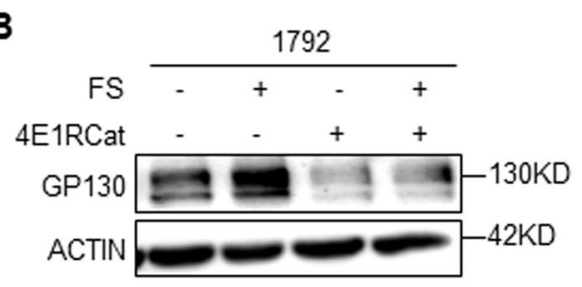

D

FS $\frac{\text { A549 }}{-+-+} \frac{1792}{-++}$

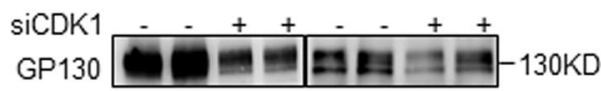

p-4E-BP1 - - - - -

$(\mathrm{T} 70)$ - 1 - $==-=$

(S65) $15 \mathrm{KD}$

4E-BP1

CDK1

ACTIN

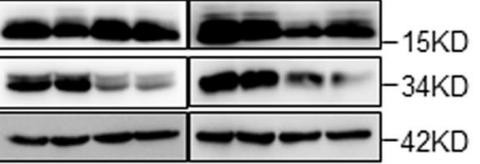

F

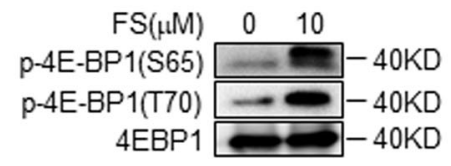

G

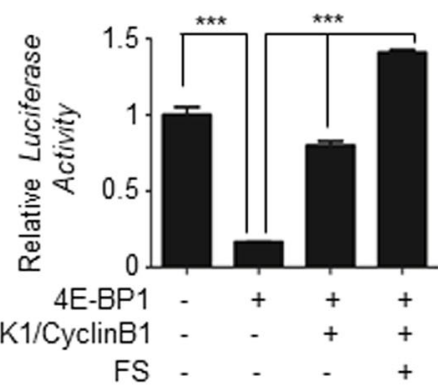

Fig. 4 (See legend on next page.) 
(see figure on previous page)

Fig. 4 Iron-dependent CDK1 promotes GP130 cap-dependent translation via 4E-BP1 phosphorylation. a, b Western blot analysis for GP130 expression in A549 or 1792 cells treated with ferrous sulfate (FS; $100 \mu \mathrm{M})$ and cap-dependent translation inhibitor 4E1RCat (50 $\mu \mathrm{M})$ for $24 \mathrm{~h}$. c Western

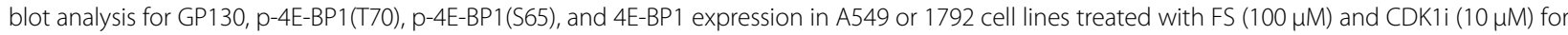
24 h. $\mathbf{d}$ Western blot analysis for GP130, p-4E-BP1(T70), p-4E-BP1(S65), and 4E-BP1 expression in A549 or 1792 transfected with siNC/siCDK1, treated with FS (100 MM) for $24 \mathrm{~h}$. e Western blot analysis for GP130, p-STAT3, p-4E-BP1(T70), p-4E-BP1(S65), and 4E-BP1 expression in A549 cells treated with CDK1i $(10 \mu \mathrm{M})$ for indicated time. $\mathbf{f}$ In vitro phosphorylation assay to assess 4E-BP1 phosphorylation. Recombinant GST-CDK1/CyclinB1 and GST-4EBP1 were added into $1 x$ protein kinase buffer in the presence 0 or $10 \mu \mathrm{M} \mathrm{FS}$, Supplemented with $200 \mu \mathrm{M} \mathrm{ATP}$, and incubated at $30^{\circ} \mathrm{C}$ for 30 min. The reactions were stopped by adding loading buffer and boiled at $95^{\circ} \mathrm{C}$ for $5 \mathrm{~min}$. Then these protein samples were subjected to western blot. $\mathbf{g} \mathrm{In}$ vitro protein translation assay. Forty microliters of TNT ${ }^{\circledR}$ Quick Master Mix, $1 \mu$ l Methionine (1 mM), $2 \mu$ l pcDNA3.1-Luciferase plasmid, and $7 \mu$ in vitro protein phosphorylation reaction products from indicated conditions, were incubated at $30^{\circ} \mathrm{C}$ for $90 \mathrm{~min}$. At last, we evaluated the luciferase translation level. $\mathbf{h}$ Schematic representation of hypophosphorylated 4E-BP1 sequestering elF4E from the elF4F cap initiation complex and turning "Off" GP130 cap-dependent translation. However, when iron binds CDK1 and enhances its activity, 4E-BP1 is hyperphosphorylated and capdependent translation is turned "On"
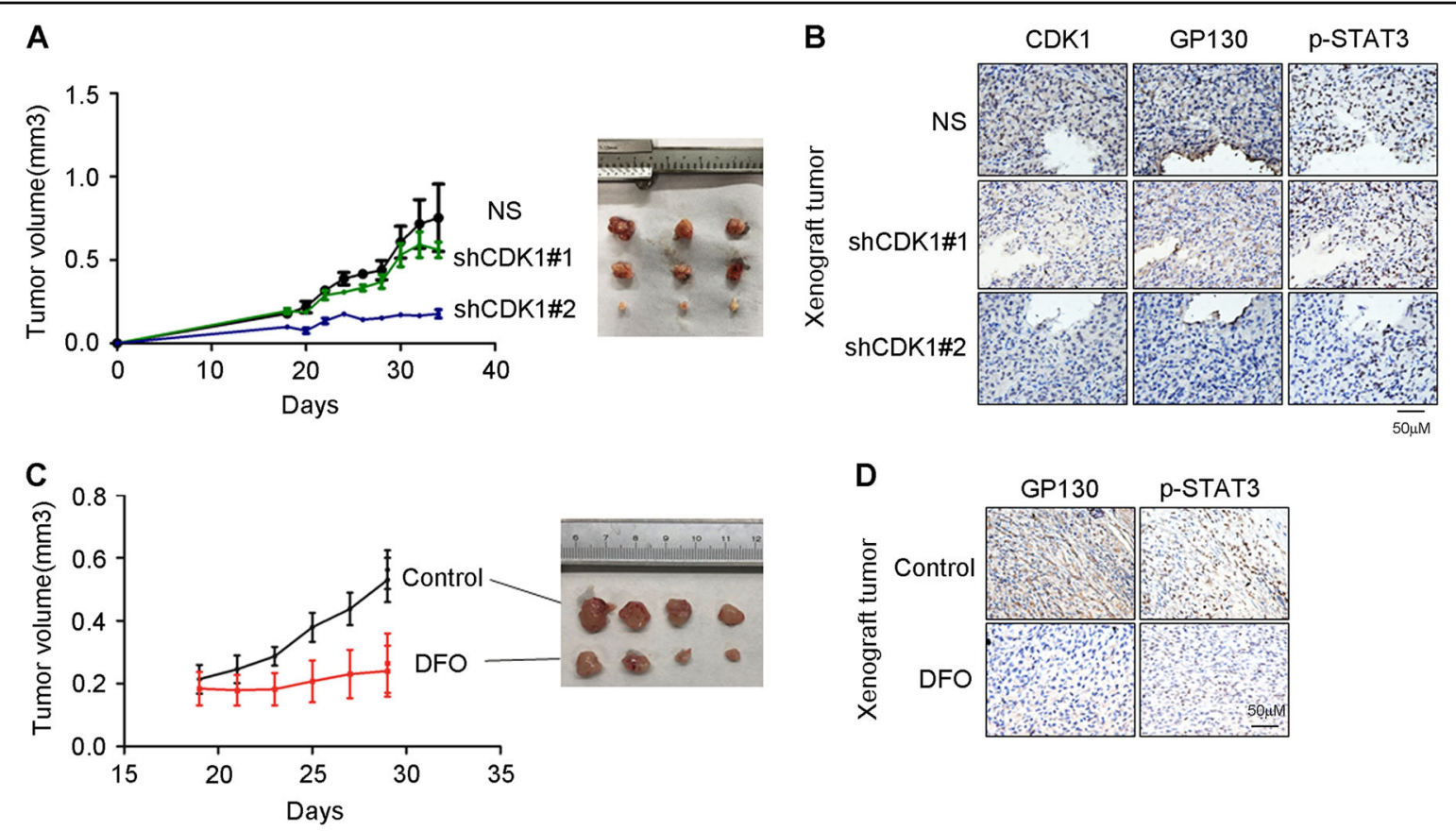

Fig. 5 CDK1 knockdown and iron chelator DFO decrease tumorigenicity and GP130/STAT3 signaling in vivo. a Nude mice were injected with A549-NS/ShCDK1 $\# 1$ and \#2) cells $\left(1 \times 10^{6}\right.$ cells). Tumor volumes were assessed in the indicated cell lines at the indicated time points. b Representative images of immunohistochemical (IHC) staining for CDK1, GP130, and p-STAT3 in A549-NS/shCDK1(\#1 and \#2) xenograft tumor tissues. c Nude mice were injected with A549 cells $\left(1.5 \times 10^{6}\right.$ cells). After 19 days, the mice were administered an intraperitoneal injection of DFO $(16 \mathrm{mg} / \mathrm{kg})$ dissolved in $0.9 \% \mathrm{NaCl}(\mathrm{DFO})$, or saline solution alone (Control), once a day for 12 days. Tumor volumes were assessed at the indicated time points. d Representative images of immunohistochemical (IHC) staining for GP130, and p-STAT3 in xenograft tumor tissues of control group (Control) or DFO group (DFO)

normal lung tissues. CDK1, GP130, and p-STAT3 were upregulated in lung cancer tissues compared with adjacent normal lung tissues (Fig. 6c). Altogether, these results suggest that elevated CDK1/GP130/ STAT3 signaling may essentially contribute to lung carcinogenesis.

\section{Discussion}

In this study, we first proposed that iron-dependent CDK1 activity upregulates GP130 and activates downstream STAT3 signaling. CDK1 and STAT3 are essential for iron-mediated colony formation. CDK1 knockdown and iron chelator DFO decrease tumorigenicity and GP130/STAT3 signaling in vivo. Moreover, CDK1/GP130/STAT3 signaling were elevated in lung cancer tissues compared with adjacent normal lung tissues.

CDK1 is a key determinant of mitotic progression, and its activity is dysregulated by direct genetic alterations during tumorigenesis ${ }^{2}$. CDK1 is upregulated in lung 


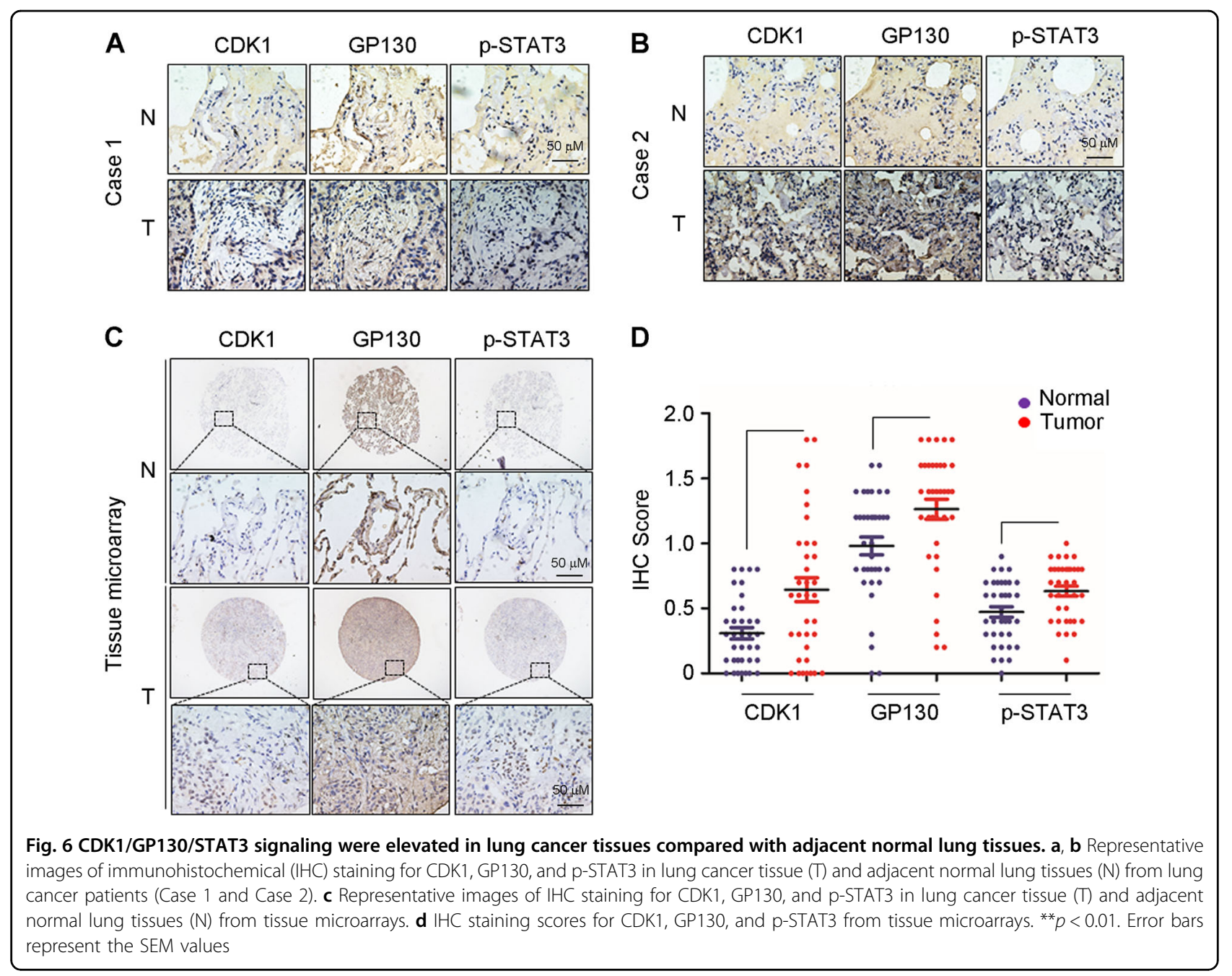

adenocarcinoma and is suggested to have a poor prognosis ${ }^{3}$. Emerging studies suggest that selective targeting of CDK1 might constitute a novel plausible strategy for tumor treatment in certain contexts ${ }^{4-6}$. However, the molecular mechanism and the potential applications of CDK1 with respect to lung cancer are yet unclear. CDK1 promotes cap-dependent translation via 4E-BP1 phosphorylation and might contribute to cell transformation $^{25,26}$. This study is the first, to our knowledge, to propose that iron promotes GP130 cap-dependent translation via CDK1-dependent 4E-BP1 phosphorylation. GP130 is an indispensable receptor of IL-6-type cytokines $^{15}$, and iron deprivation- or CDK1 inhibitionmediated GP130 downregulation markedly blocked STAT3 signaling. IL-6-type cytokines are the critical lynchpins between inflammation and cancer ${ }^{16}$. Systemic and pulmonary IL- 6 are elevated in lung adenocarcinoma patients and are related to poor patient survival ${ }^{17-20}$. IL-6 promotes KRAS-driven lung carcinogenesis, and genetic ablation of either IL-6 or STAT3 suppresses the extent of lung cancer ${ }^{21}$. In addition, lung cancer chemosensitivity is reportedly associated with STAT3 signaling ${ }^{28,29}$. Altogether, the present results suggest a potential preventive and treatment strategy for lung cancer via suppression of GP130/STAT3 signaling via CDK1 inhibition or iron deprivation.

Although the present results support the premise that STAT3 signaling promotes lung carcinogenesis, STAT3 reportedly unexpectedly opposed tumorigenesis in KRAS-mutant lung adenocarcinoma, lung epithelialspecific inactivation of Stat3 promoted Kras ${ }^{\mathrm{G} 12 \mathrm{D}}$-driven lung adenocarcinoma ${ }^{30}$. The tumor-suppressive role of STAT3 is attributed to its ability to inhibit NF-kBinduced transcription of the proangiogenic chemokine Cxcl1, thereby suppressing tumor vascularization and tumor progression ${ }^{30}$. Furthermore, STAT3 promotes lung carcinogenesis via IL-6 trans-signaling without altering Cxcl1 transcription; hence, these results were considered to probably be caused by different STAT3 upstream cytokines, or that STAT3 heterodimers 


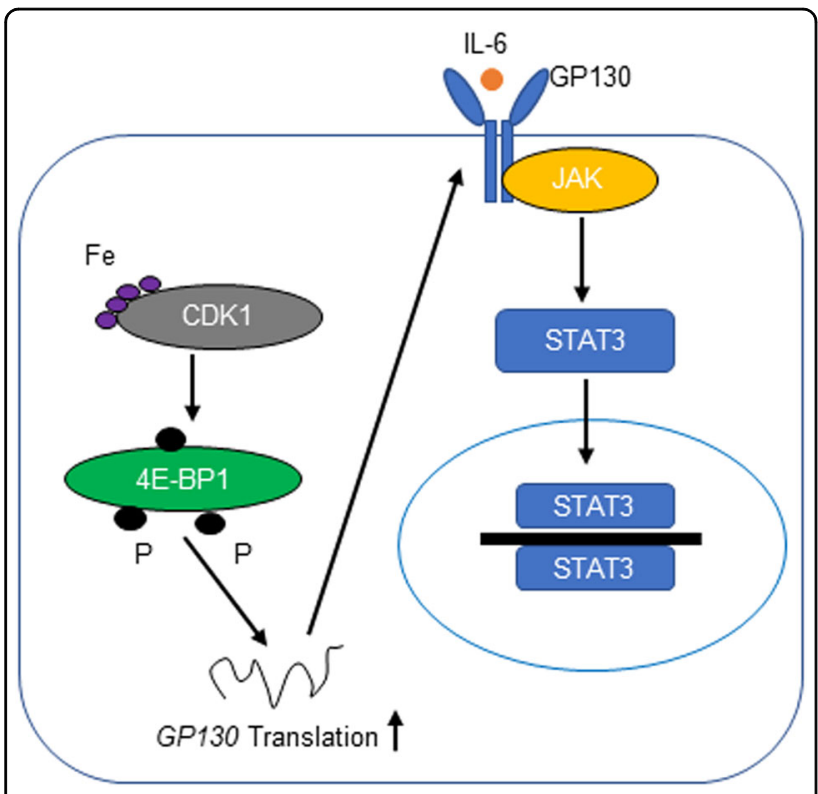

Fig. 7 Schematic representation of the mechanism underlying the role of iron in JAK/STAT3 signaling in lung cancer tissue. Iron binds CDK1 and enhances its activity to phosphorylate 4E-BP1, which in turn increases GP130 cap-dependent translation and downstream JAK/STAT3 signaling upon IL-6 binding

modulated a different network of gene targets ${ }^{21}$. In the present study, iron deprivation or CDK1 inhibition downregulated IL-6-type cytokine receptor GP130, which theoretically blocked both binding of STAT3 upstream cytokines and downstream STAT3 heterodimers signaling.

Epidemiological and experimental data suggest that iron contributes to lung carcinogenesis ${ }^{8-14}$; however, the underlying mechanism is still unknown. Iron reportedly binds CDK1 directly and enhances its activity, CDK1 phosphorylates JAK1 directly, and the activation of JAK/ STAT3 signaling promotes colorectal cancer ${ }^{9}$. In the present study, CDK1 upregulated GP130, which then served as the upstream receptor of JAK. This seemingly complements the mechanism underlying CDK1-mediated activation of STAT3 signaling. It was first proposed that iron-dependent CDK1 activity activates GP130/STAT3 signaling to promote lung carcinogenesis. As an important trace element, iron is required for protein and enzyme activity and is closely associated with ferroptosis, a form of cell death caused by the iron-mediated lipid peroxide generation ${ }^{8}$. However, numerous questions regarding the mechanism of action and application of iron in lung cancer remain unanswered.

In conclusion, the present results indicate that iron binds CDK1, thereby enhancing 4E-BP1 phosphorylation, subsequently increasing GP130 cap-dependent translation and downstream JAK/STAT3 signaling upon IL-6 binding (Fig. 7). The present results further the current understanding of the molecular mechanism underlying the role of iron and CDK1 in lung carcinogenesis.

\section{Materials and methods \\ Cell lines and cell culture}

Human lung adenocarcinoma cell lines A549 and 1792 were obtained from ATCC, and cultured in RPMI 1640 medium supplemented with $10 \%$ fetal bovine serum. Human embryonic kidney cells HEK293T from ATCC were cultured in DMEM medium supplemented with 10\% fetal bovine serum. These cells were characterized/ authenticated by DNA typing at Shanghai Jiao Tong University Analysis Core.

\section{Transfection and stable cell lines generation}

GP130 and CDK1 siRNA were purchased from Biomics Biotech. CDK1 and STAT3 shRNA were obtained from Shanghai Jiao Tong University cDNA library. Nonspecific shRNA (NS) was used as control. HEK293T cells were transfected with lenti-shRNA, and the lentiviruses-containing media were harvested after $48 \mathrm{~h}$ and used to infect A549 and 1792. All transfections were performed with Lipofectamine 2000 (Invitrogen) according to the manufacturer's protocols. Stably transfected cells were selected with puromycin $(2 \mu \mathrm{g} / \mathrm{ml})$.

\section{Soft-agar colony formation assay}

Melted 2\% agar solution combined with indicated conditional medium (RPMI 1640 supplemented with $0 \mu \mathrm{M}$ or $100 \mu \mathrm{M} F S)$ at a ratio of $1: 3(\mathrm{v} / \mathrm{v})$ and added into 24-well plates. Plates were placed at $4{ }^{\circ} \mathrm{C}$ in refrigerator for $10 \mathrm{~min}$ to solidify. Thereafter, Cell suspensions $(500 / 50 \mu \mathrm{l})$ were pipetted onto the solidified agar. Top agar layer was added over the cell suspensions, consisting of the $2 \%$ agar solution combined with indicated conditional medium $(1: 6 \mathrm{v} / \mathrm{v})$ along with matrigel $(1: 30 \mathrm{v} / \mathrm{v})$. The established colonies number were counted after 2 weeks.

\section{Sphere culture assay}

A549 and 1792 cells were seeded into low-adherent 96well plates (2000 per well), and were cultured in indicated conditional medium. The culture media were replaced every 3 days. After 12 days, the spheres were observed and photographed.

\section{Western blot}

Cells were washed twice by PBS (Hyclone), and then lysed with RIPA buffer $(150 \mathrm{mM} \mathrm{NaCl}, 50 \mathrm{mM}$ Tris, $25 \mathrm{mM} \mathrm{NaF}, 2 \mathrm{mM}$ Na3VO4, $0.5 \%$ deoxycholate, $1 \%$ NP40) supplement with protease inhibitor cocktail (biomark.cn). Equal amount of protein samples was mixed with loading buffer and boiled at $95^{\circ} \mathrm{C}$ for $5 \mathrm{~min}$. Then proteins were separated by SDS-PAGE and transferred to nitrocellulose $(\mathrm{NC})$ membranes. Five percent non-fat milk 
were used to block non-specific binding sites of the membranes. After that, primary antibody was added on the membranes and incubated at $4{ }^{\circ} \mathrm{C}$ overnight. Membranes were washed three times by TBST, then secondary antibody was incubated at room temperature for $1 \mathrm{~h}$. ECL kit (Millipore) was used for Chemiluminescence ${ }^{31}$. CDK1 (sc-8395, sc-137035) and GP130 (sc-655) primary antibody were purchased from Santa cruz. p-STAT3 (\#9145), STAT3 (\#9139), 4E-BP1 (\#9644), p-4E-BP1 Ser65 (\#9451), and p-4E-BP1 Thr70 (\#9455) were purchased from Cell signaling technology. HRP-ACTIN (HRP-60008) was purchased from proteintech.

\section{Protein stability}

A549 cells was seeded into 6-well plates. At the beginning of each chase, cycloheximide (CHX, $30 \mu \mathrm{g} / \mathrm{ml})$ was added to block translation. CDK1i $(10 \mu \mathrm{M})$ was added at the beginning of each chase. FS $(100 \mu \mathrm{M})$ pre-treatment was administered for $24 \mathrm{~h}$. Cells were harvested at the indicated time point. Western blot analysis for GP130 expression and the relative amounts of GP130 were calculated after normalization (GP130/ACTIN).

\section{Quantitative real-time PCR}

Total RNA was extracted from cells using the RNA simple Total Kit (TIANGEN). cDNA was synthesized using FastQuant RT kit(TIANGEN). Furthermore, Quantitative real-time PCR experiments were using SuperReal PreMix Plus SYBR Green kit (TIANGEN). ${ }^{31}$ GP130 mRNA levels were measured using the primer (forward: 5'-CGGACAGCTTGAACAGAATGT$3^{\prime}$ and reverse: 5'-ACCATCCCACTCACACCTCA-3'), GAPDH (forward: 5' -TGCACCACCAACTGCTTAGC-3' and reverse: 5'-GGCATGGACTGTGGTCATGAG-3').

\section{In vitro protein phosphorylation assay and in vitro protein translation assay}

One-hundred nanograms of recombinant GST-CDK1/ CyclinB1 (SRP5009, Sigma-Aldrich) and $0.2 \mu \mathrm{g}$ recombinant GST-4E-BP1 (Ag5056, Proteintech) were added into $24 \mu \mathrm{l} 1 \mathrm{x}$ protein kinase buffer (\#B6022, New England Biolabs) in the presence or absence of $10 \mu \mathrm{M}$ FS, Supplemented with $200 \mu \mathrm{M}$ ATP (P0756, New England Biolabs), and incubated at $30^{\circ} \mathrm{C}$ for $30 \mathrm{~min}$. The reactions were stopped by adding loading buffer and boiled at $95^{\circ} \mathrm{C}$ for $5 \mathrm{~min}$. Then these protein samples were subjected to western blot. In vitro protein translation assay was performed with $\mathrm{TnT}^{\circledast}$ Quick Coupled Transcription/Translation System (L1170; Promega) according to the manufacturer's protocols. Forty microliters $\mathrm{TNT}^{\circledR}$ Quick Master Mix, $1 \mu \mathrm{l}$ Methionine $(1 \mathrm{mM}), 2 \mu \mathrm{l}$ pcDNA3.1Luciferase plasmid, and $7 \mu \mathrm{l}$ in vitro protein phosphorylation reaction products from indicated conditions, were incubated at $30^{\circ} \mathrm{C}$ for $90 \mathrm{~min}$. At last, we evaluated the luciferase translation level via detecting the light intensity upon addition of luciferin.

\section{Tumorigenicity in nude mice}

Human lung adenocarcinoma cell lines A549 were stably transfected. Then A549-NS/A549-shCDK1 cells $\left(1 \times 10^{6}\right)$ combined with matrigel were injected subcutaneously into 8-week-old nude mice. The tumor volume was measured every 2 or 3 days. Nude mice were injected with A549 cells $\left(1.5 \times 10^{6}\right.$ cells) combined with Matrigel. After 19 days, the mice were administered an intraperitoneal injection of DFO $(16 \mathrm{mg} / \mathrm{kg})$ dissolved in $0.9 \% \mathrm{NaCl}$, or saline solution alone, once a day for 12 days. Tumor volumes were measured every 2 days. All mice were maintained according to a protocol approved by Shanghai Jiao Tong University, School of Medicine Animal Care and Use Committee [experimental animal use permission No: SYXK (Shanghai) 2008-0050] in the specific pathogen-free animal facility in the university.

\section{Immunohistochemistry (IHC)}

NSCLC patients tissue samples obtained from the Second Affiliated Hospital of Dalian Medical University (Dalian, China) $^{32}$. Tissue microarray presented from Jiong Deng's Lab. Tissue samples were stained to identify CDK1, GP130, and p-STAT3 proteins. The IHC protocol and score method were performed as previously descri$\operatorname{bed}^{33}$. All antibodies were diluted for use according to the manufacturer's instructions.

\section{Statistical analyses}

Data were analyzed using the software GraphPad Prism Version 5.01. Data are presented as the mean \pm SD. A two-tailed unpaired $t$-test was used to compare results. $p<0.05$ was considered statistically significant.

\section{Acknowledgements}

This study was supported in part by grants from the National Natural Science Foundation of China 81330060, 81620108022, 91129303, 91729302, 81572759, the National High Technology Research and Development Program (863 Program Projects) of China (\#2015AA020409), the Opening Project of Zhejiang Provincial Top Key Discipline of Clinical Medicine (No. LKFyc05), Special Grant for Translational Medicine Dalian Medical University (2015001), Ministry of Science and Technology No. 2013CB910901, the Key Discipline Construction Project of Pudong Health Bureau of Shanghai (PWZxk2017-09) and Municipal Human Resources Development Program for Outstanding Leaders in Medical Disciplines in Shanghai (2017BR032).

\section{Author details}

'Department of Respiratory Medicine, The Second Affiliated Hospital, Dalian Medical University, Dalian, China. ${ }^{2}$ Key Laboratory of Cell Differentiation and Apoptosis of Chinese Minister of Education, Shanghai, China. ${ }^{3}$ Department of Laboratory Medicine, Shanghai East Hospital, Tongji University School of Medicine, Shanghai, China. ${ }^{4}$ Department of Oncology, Shanghai General Hospital, Shanghai Jiao Tong University School of Medicine, Shanghai, China. ${ }^{5}$ Department of Health Examination Center, The Second Affiliated Hospital, Dalian Medical University, Dalian, China. ${ }^{6}$ Department of Scientific Research Center, The Second Affiliated Hospital, Dalian Medical University, Dalian, China. ${ }^{7}$ Department of Pharmacy, The Second Affiliated Hospital, Dalian Medical University, Dalian, China 


\section{Authors' contributions}

Y.K., W.G., and J.L. performed the experiments and were assisted by D.X., Y.L., H. Z., X.D., H.W., M.X., H.S., T.W., B.J., K.L., M.H., and W.W. D.X. provided experiments about animal. Y.L. constructed the plasmids. H.Z. and M.X. provided human lung tissue samples. Y.K., Q.W., and J.D. designed research, and wrote the manuscript.

\section{Conflict of interest}

The authors declare that they have no conflict of interest.

\section{Publisher's note}

Springer Nature remains neutral with regard to jurisdictional claims in published maps and institutional affiliations.

Supplementary Information accompanies this paper at (https://doi.org/ 10.1038/s41419-019-1528-y).

Received: 24 October 2018 Revised: 8 March 2019 Accepted: 12 March 2019

Published online: 01 April 2019

\section{References}

1. Siegel, R. L., Miller, K. D. \& Jemal, A. Cancer statistics, 2018. CA Cancer J. Clin. 68, 7-30 (2018).

2. Asghar, U., Witkiewicz, A. K., Turner, N. C. \& Knudsen, E. S. The history and future of targeting cyclin-dependent kinases in cancer therapy. Nat. Rev. Drug. Discov. 14, 130-146 (2015).

3. Shi, Y. X. et al. Prognostic and predictive values of CDK1 and MAD2L1 in lung adenocarcinoma. Oncotarget 7, 85235 (2016).

4. Kang, J., Sergio, C. M., Sutherland, R. L. \& Musgrove, E. A. Targeting cyclindependent kinase 1 (CDK1) but not CDK4/6 or CDK2 is selectively lethal to MYC-dependent human breast cancer cells. BMC Cancer 14, 32-32 (2014).

5. Johnson, N. et al. Compromised CDK1 activity sensitizes BRCA-proficient cancers to PARP inhibition. Nat. Med. 17, 875-882 (2011).

6. Wu, C. X. et al. Blocking CDK1/PDK1/beta-Catenin signaling by CDK1 inhibitor RO3306 increased the efficacy of sorafenib treatment by targeting cancer stem cells in a preclinical model of hepatocellular carcinoma. Theranostics $\mathbf{8}$ 3737-3750 (2018).

7. Arredondo, M. \& Nunez, M. T. Iron and copper metabolism. Mol. Asp. Med. 26 313-327 (2005).

8. Torti, S. V., Manz, D. H., Paul, B. T., Blanchette-Farra, N. \& Torti, F. M. Iron and cancer. Annu. Rev. Nutr. 38, 97-125 (2018).

9. Xue, X. et al. Iron uptake via DMT1 integrates cell cycle with JAK-STAT3 signaling to promote colorectal tumorigenesis. Cell Metab. 24, 447-461 (2016).

10. Hann, H. W., Stahlhut, M. W. \& Menduke, H. Iron enhances tumor growth. Observation on spontaneous mammary tumors in mice. Cancer 68, 2407 (1991).

11. Hann, H. W. L., Stahlhut, M. W. \& Blumberg, B. S. Iron nutrition and tumor growth: decreased tumor growth in iron-deficient Mice. Cancer Res. 48, 4168-4170 (1988)
12. Lui, G. Y. et al. The iron chelator, deferasirox, as a novel strategy for cancer treatment: oral activity against human lung tumor xenografts and molecular mechanism of action. Mol. Pharmacol. 83, 179-190 (2013).

13. Cloonan, S. M. et al. Mitochondrial iron chelation ameliorates cigarette smoke-induced bronchitis and emphysema in mice. Nat. Med. 22, 163-174 (2016).

14. Singer, J. P., Lederer, D. J. \& Baldwin, M. R. Frailty in pulmonary and critical care medicine. Ann. Am. Thorac. Soc. 13, 1394 (2016).

15. Heinrich, P. C. et al. Principles of interleukin (IL)-6-type cytokine signalling and its regulation. Biochem. J. 374(Pt 1), 1-20 (2003).

16. Taniguchi, K. \& Karin, M. IL-6 and related cytokines as the critical lynchpins between inflammation and cancer. Semin. Immunol. 26, 54-74 (2014).

17. Yanagawa, $H$. et al. Serum levels of interleukin 6 in patients with lung cancer. Br. J. Cancer 71, 1095-1098 (1995).

18. Gao, S. P. et al. Mutations in the EGFR kinase domain mediate STAT3 activation via IL-6 production in human lung adenocarcinomas. (Research article)(epidermal growth factor receptor)(signal transducer and activator of transcription 3)(interleukin-6). J. Clin. Investig. 117, 3846-3856 (2008).

19. Yeh, H., Lai, W., Chen, H., Liu, H. \& Su, W. Autocrine IL-6-induced Stat3 activation contributes to the pathogenesis of lung adenocarcinoma and malignant pleural effusion. Oncogene 25, 4300-4309 (2006).

20. Haura, E. B., Livingston, S. \& Coppola, D. Autocrine interleukin-6/interleukin-6 receptor stimulation in non-small-cell lung cancer. Clin. Lung. Cancer $\mathbf{7}$ 273-275 (2006).

21. Brooks, G. D. et al. IL6 Trans-signaling promotes KRAS-driven lung carcinogenesis. Cancer Res. 76, 866-876 (2016).

22. Miller, A. et al. Blockade of the $I L-6$ trans-signalling/STAT3 axis suppresses cachexia in Kras-induced lung adenocarcinoma. Oncogene 36, 3059-3066 (2017).

23. Dixon, S. J. \& Stockwell, B. R. The role of iron and reactive oxygen species in cell death. Nat. Chem. Biol. 10, 9 (2014).

24. Carballo, M. et al. Oxidative stress triggers STAT3 tyrosine phosphorylation and nuclear translocation in human lymphocytes. J. Biol. Chem. 274, 17580-17586 (2012).

25. Shuda, M. et al. CDK1 substitutes for mTOR kinase to activate mitotic capdependent protein translation. Proc. Natl Acad. Sci. USA 112, 5875-5882 (2015).

26. Velásquez, $C$. et al. Mitotic protein kinase CDK1 phosphorylation of mRNA translation regulator 4E-BP1 Ser83 may contribute to cell transformation. Proc. Natl Acad. Sci. USA 113, 8466 (2016).

27. Yanagiya, A. et al. Translational Homeostasis via the mRNA Cap-Binding Protein, elF4E. Mol. Cell 46, 847-858 (2012).

28. Maryam, A. et al. Alantolactone induces apoptosis, promotes STAT3 glutathionylation and enhances chemosensitivity of A549 lung adenocarcinoma cells to doxorubicin via oxidative stress. Sci. Rep. 7, 6242 (2017).

29. MacDonagh, L. et al. BBl608 inhibits cancer stemness and reverses cisplatin resistance in NSCLC. Cancer Lett. 428, 117-126 (2018).

30. Grabner, B. et al. Disruption of STAT3 signalling promotes KRAS-induced lung tumorigenesis. Nature. Communications 6, 6285 (2015).

31. Song, $H$. et al. GPRC5A deficiency leads to dysregulated MDM2 via activated EGFR signaling for lung tumor development. Int. J. Cancer 144, 777-787 (2019).

32. Zhao, H. et al. CD47 promotes tumor invasion and metastasis in non-small cell lung cancer. Sci. Rep. 6, 29719 (2016).

33. Sun, B. et al. Gprc5a-knockout mouse lung epithelial cells predicts ceruloplasmin, lipocalin 2 and periostin as potential biomarkers at early stages of lung tumorigenesis. Oncotarget 8, 13532 (2017). 